\title{
Variabilidade de micronutrientes, matéria orgânica e argila de um Latossolo submetido a sistemas de preparo(1)
}

\author{
Pedro Marques da Silveira(2) e Adriany Alves da Cunha ${ }^{(3)}$
}

\begin{abstract}
Resumo - O objetivo deste trabalho foi estudar os efeitos dos sistemas de preparo do solo, arado de aiveca, grade aradora e plantio direto, sobre a variabilidade de atributos físico-químicos do solo. O trabalho foi conduzido em Latossolo Vermelho perférrico, textura argilosa, cultivado com milho no verão e feijão no inverno, durante cinco anos consecutivos. As amostras de solo foram coletadas em 49 pontos, de uma malha quadrada de $7 \times 7$, espaçados de $4 \times 4 \mathrm{~m}$, nas profundidades de $0-5 \mathrm{~cm}$ e $5-20 \mathrm{~cm}$ de solo. Os valores de $\mathrm{Cu}, \mathrm{Zn}, \mathrm{Fe}, \mathrm{Mn}, \mathrm{B}$, matéria orgânica (MO) e argila do solo variaram nos diferentes tratamentos. Na profundidade de $0-5 \mathrm{~cm}$, os valores de $\mathrm{Cu}, \mathrm{Mn}, \mathrm{B}, \mathrm{MO}$ e argila foram maiores no sistema plantio direto. Os valores de $\mathrm{Cu}$ e de $\mathrm{Zn}$ apresentaram as maiores variabilidades, e os de $\mathrm{MO}$ e argila, as menores. Utilizando-se o procedimento de coletar 20 subamostras para formar uma amostra composta, os teores de argila, MO, B e Mn estariam sendo estimados com erro em torno de $10 \%$ do valor médio. Para o $\mathrm{Cu}$ e o $\mathrm{Zn}$ a variação em torno da média atingiu mais de $25 \%$.
\end{abstract}

Termos para indexação: arado de aiveca, grade de disco, plantio direto, fertilidade do solo.

Variability of micronutrients, organic matter and clay content in Oxisol under different tillage systems

\begin{abstract}
The objective of this study was to determine the effect of soil tillage systems, moldboard plough, harrow disc and no-tillage, on the variability of some soil physico-chemical properties. The study was conducted in a clayey Oxisol, subjected to different soil tillage systems for five consecutive years and cultivated with corn in the summer and bean in the winter. Forty nine soil samples were collected from a grid 7x7 lattice sampling area spaced $4 \times 4 \mathrm{~m}$ at $0-5 \mathrm{~cm}$ and $5-20 \mathrm{~cm}$ soil depth. The values of $\mathrm{Cu}, \mathrm{Zn}, \mathrm{Fe}, \mathrm{Mn}, \mathrm{B}$, organic matter and clay contents of soil varied for different treatments. The $\mathrm{Cu}, \mathrm{Mn}, \mathrm{B}$, organic matter and clay content values were higher in the $0-5 \mathrm{~cm}$ soil depth in no-tillage treatment. Among the soil chemical properties evaluated, the concentrations of $\mathrm{Cu}$ and $\mathrm{Zn}$ showed the highest variability, whereas organic matter and clay contents had the lowest. In 20 samples analyzed, the variables clay, organic matter, B and Mn were estimated with an error of $10 \%$ of the actual value. For $\mathrm{Cu}$ and $\mathrm{Zn}$, the mean variation using the same procedure reached over $25 \%$.
\end{abstract}

Index terms: mouldboard ploughs, disc harrows, direct sowing, soil fertility.

\section{Introdução}

Numa área cultivada existem fontes adicionais de heterogeneidade no solo, por causa do manejo exercido pelo homem nas suas mais variadas formas. Os sistemas de preparo do solo alteram os atributos físico-químicos do solo, por promoverem o revolvimento da camada explorada pelas raízes e o acúmulo

\footnotetext{
(1) Aceito para publicação em 28 de fevereiro de 2002.

(2) Embrapa-Centro Nacional de Pesquisa de Arroz e Feijão, Caixa Postal 179, CEP 75375-000 Santo Antônio de Goiás, GO. E-mail: pmarques@cnpaf.embrapa.br

(3) Universidade Federal de Goiás, Caixa Postal 31, CEP 74001-970 Goiânia, GO. E-mail: adrianycunha@bol.com.br
}

de material orgânico, interferindo, assim, diretamente na distribuição dos nutrientes no perfil do solo. A adoção do sistema plantio direto vem expandindo-se na Região Central do Brasil, é uma alternativa ao sistema convencional de preparo do solo, e pode contribuir para a sustentabilidade de sistemas agrícolas intensivos. Souza (1992) pressupôs que o plantio direto apresentasse maior variabilidade nos teores de matéria orgânica e de nutrientes no perfil do solo, do que o sistema convencional, em decorrência do não-revolvimento da camada superficial do solo.

Couto \& Klamt (1999), em trabalho sobre a variabilidade espacial de micronutrientes no solo, verifi- 
caram que a deficiência do micronutriente Mn numa área do experimento refletiu-se diretamente na produtividade de grãos de milho cultivado naquela área.

A variabilidade espacial dos micronutrientes e também de outras características físicas e químicas do solo determina a intensidade e a forma de amostragem para compor amostras representativas desse solo. Para uma amostragem representativa da fertilidade de uma área, é necessário o conhecimento da variabilidade desses atributos físicos e químicos.

O número mínimo de amostras de solo para estimar o valor médio da variável de interesse com uma determinada exatidão tem sido calculado em vários trabalhos. Segundo Souza (1992), o procedimento consiste em coletar ao acaso certo número de amostras individuais, analisá-las e calcular os coeficientes de variação dos dados, achar os valores de Tabela do teste $\mathrm{t}$ correspondentes ao número de graus de liberdade do quadrado médio residual, estabelecer a diferença permitida em torno da média e, assim, calcular o número mínimo de amostras individuais a serem coletadas em futuras amostragens.

O número de 15 a 20 subamostras simples por amostra composta, recomendada para amostragem de glebas de propriedades agrícolas, é um compromisso entre o ótimo teórico, considerando as determinações de maior erro, e o que é exequíivel na prática (Raij, 1991; Raij \& Bataglia, 1991; Comissão de Fertilidade do Solo-RS/SC, 1995). Segundo Santos \& Vasconcellos (1987), o número de amostras para análise química que represente bem uma área pode variar com o manejo do solo, cultura anterior e fertilização da área.

O objetivo deste trabalho foi avaliar o efeito de sistemas de preparo do solo com arado de aiveca, grade aradora e plantio direto na variabilidade, na distribuição espacial e no número de amostras necessárias para a avaliação dos teores de $\mathrm{Cu}, \mathrm{Zn}, \mathrm{Fe}$, Mn e B, da matéria orgânica e de argila em um Latossolo Vermelho perférrico.

\section{Material e Métodos}

O trabalho foi conduzido em um Latossolo Vermelho perférrico, textura argilosa, na Fazenda Capivara, da Embrapa-Centro Nacional de Pesquisa de Arroz e Feijão, latitude $16^{\circ} 28^{\prime} \mathrm{S}$ e longitude $49^{\circ} 17^{\prime} \mathrm{O}$, em Santo Antônio de Goiás, GO. A área foi cultivada durante cinco anos consecutivos, 1992 a 1996, com as culturas de milho no verão e feijão no inverno, sob irrigação por aspersão, sistema pivô central. As culturas foram semeadas em solo sob os sistemas de preparo com arado de aiveca, com grade aradora e plantio direto.

No primeiro tratamento, usou-se um arado de três aivecas, de 30,5 cm de largura, incorporando-se ao solo os resíduos das culturas até a profundidade de $30 \mathrm{~cm}$, seguindo-se de uma gradagem com grade leve; no segundo, usouse uma grade pesada de 20 discos, de $66 \mathrm{~cm}$ de diâmetro, incorporando ao solo os resíduos até $15 \mathrm{~cm}$, e no terceiro, o plantio direto foi realizado com semeadora-adubadora apropriada, permanecendo na superfície do solo o resíduo das culturas. A semeadora-adubadora utilizada era provida de disco de corte de palhada, de sulcador de haste para adubação, e de disco duplo desencontrado, para semeadura. Por ocasião do plantio de cada cultura, aplicaram-se $400 \mathrm{~kg} \mathrm{ha}^{-1}$ de adubo, da fórmula 5-30-15.

No quarto ano de plantio, foram aplicadas $2,5 \mathrm{t} \mathrm{ha}^{-1} \mathrm{de}$ calcário em toda a área experimental. Nos tratamentos arado e grade, o corretivo foi incorporado ao solo, e no plantio direto permaneceu na superfície. A precipitação pluvial ocorrida nos anos de 1992, 1993, 1994, 1995 e 1996 foi de $1.521,1.116,1.676,1.487$ e $1.145 \mathrm{~mm}$, respectivamente, concentrados principalmente nos meses de outubro a março.

As amostras para as análises físico-químicas foram coletadas, em outubro de 1996, em todos os tratamentos, em 49 pontos de uma malha quadrada de $7 \times 7$, com espaçamento de $4 \times 4 \mathrm{~m}$, nas profundidades de $0-5 \mathrm{~cm}$ e $5-20 \mathrm{~cm}$. A coleta em profundidade teve como objetivo a avaliação do acúmulo superficial dos nutrientes. Nas amostras foram determinados os teores de $\mathrm{Cu}, \mathrm{Zn}$, $\mathrm{Fe}, \mathrm{Mn}, \mathrm{B}$, de matéria orgânica (MO) e de argila do solo, conforme Embrapa (1997). Os dados foram analisados calculando-se os valores médio, mínimo e máximo e os coeficientes de variação (CV). Os valores médios das variáveis foram comparados pelo teste $t$.

Foram determinados, também, os números de subamostras necessárias para formar uma amostra composta e estimar o valor médio das variáveis, usando a fórmula descrita por Cline (1944):

$\mathrm{N}=(\mathrm{T} \alpha \times \mathrm{CV})^{2} / \mathrm{D}^{2}$, em que N é o número mínimo de amostras; T $\alpha$ é o valor do teste t de Student para o nível de probabilidade de 95\%; CV é o coeficiente de variação; $D$ é a porcentagem de variação em torno da média $(5,10,15,20,25$ e $30 \%)$.

Foi realizada, também, a espacialização dos dados de campo, de Zn e MO do solo, utilizando o Sistema de Informações Geográficas desenvolvido pelo Instituto Nacional de Pesquisas Espaciais (1990). 


\section{Resultados e Discussão}

Quanto ao $\mathrm{Cu}$ houve diferença significativa entre os tratamentos nas duas profundidades estudadas, sendo que, os maiores valores ocorreram nos tratamentos grade e plantio direto (Tabelas 1 e 2). $\mathrm{O}$ tratamento com grade, em ambas as profundidades, não diferiu do tratamento plantio direto. De acordo com as médias observadas, os valores aumentaram na profundidade de $5-20 \mathrm{~cm}$ em relação à profundidade de 0-5 cm. Alguns trabalhos corroboram estes resultados, ou seja, aumento da concentração de $\mathrm{Cu}$ nos horizontes mais profundos do solo, simultaneamente ao aumento dos teores de argila (Horowitz \& Dantas, 1973).

Os coeficientes de variação foram relativamente altos nesta variável, em todos os tratamentos. O maior coeficiente de variação foi obtido no plantio direto, o que evidencia a alta variabilidade desse nutriente. A maior amplitude constatada no plantio direto explica o maior coeficiente de variação neste tratamento em relação aos demais. Em razão do não-revolvimento do solo, como ocorre no plantio direto, es- pera-se maior variabilidade dos dados neste tratamento.

Segundo Souza (1992), o sistema convencional com grade aradora, por revolver o solo, apresenta menor variabilidade, horizontal e vertical, quanto aos teores de matéria orgânica e nutrientes na camada superficial do solo em relação à escarificação e ao plantio direto.

Com relação ao $\mathrm{Zn}$, houve diferença significativa entre os três tratamentos na profundidade de $0-5 \mathrm{~cm}$. Na profundidade de 5-20 cm, os tratamentos arado e grade mostraram-se significativamente diferentes, mas nenhum deles diferiu do tratamento plantio direto. O tratamento arado apresentou o menor valor médio nas duas profundidades quanto a esta variável, como decorrência do revolvimento do solo provocado pela ação do implemento, e causou diluição do nutriente pela mistura com maior volume de solo.

Tanto a amplitude quanto o coeficiente de variação foram maiores para o $\mathrm{Zn}$ em relação ao cobre. $\mathrm{O} Z \mathrm{Zn}$ pode apresentar-se em maior concentração na camada superficial ou ao longo do perfil, variando de acordo com o solo. O plantio direto, na profundi-

Tabela 1. Valores médios, mínimos, máximos, coeficiente de variação (CV) e número mínimo de subamostras necessário para estimar os teores de $\mathrm{Cu}, \mathrm{Zn}, \mathrm{Fe}, \mathrm{Mn}, \mathrm{B}$, matéria orgânica (MO) e argila do solo, para várias porcentagens de variação em torno da média, nos tratamentos arado, grade e plantio direto, na profundidade de $0-5 \mathrm{~cm}$ de solo.

\begin{tabular}{|c|c|c|c|c|c|c|c|c|c|c|c|}
\hline \multirow[t]{2}{*}{ Variável } & \multirow[t]{2}{*}{ Tratamento } & \multirow[t]{2}{*}{ Média $^{(1)}$} & \multirow[t]{2}{*}{ Mínimo } & \multirow[t]{2}{*}{ Máximo } & \multirow[t]{2}{*}{$\mathrm{CV}$} & \multicolumn{6}{|c|}{ Número de subamostras para variação em torno da média } \\
\hline & & & & & & $5 \%$ & $10 \%$ & $15 \%$ & $20 \%$ & $25 \%$ & $30 \%$ \\
\hline \multirow[t]{3}{*}{$\mathrm{Cu}\left(\mathrm{mg} \mathrm{L}^{-1}\right)$} & Arado & $2,3 b$ & 1,9 & 3,7 & 15,3 & 38 & 9 & 4 & 2 & 2 & 1 \\
\hline & Grade & $2,8 \mathrm{a}$ & 2,0 & 5,6 & 20,0 & 64 & 16 & 7 & 4 & 3 & 2 \\
\hline & P. direto & $2,7 \mathrm{ab}$ & 1,5 & 7,9 & 47,9 & 367 & 92 & 41 & 23 & 15 & 10 \\
\hline \multirow[t]{3}{*}{$\mathrm{Zn}\left(\mathrm{mg} \mathrm{L}^{-1}\right)$} & Arado & $4,9 \mathrm{c}$ & 1,3 & 17,6 & 52,9 & 449 & 112 & 50 & 28 & 18 & 12 \\
\hline & Grade & $7,6 b$ & 4,7 & 12,9 & 19,8 & 62 & 16 & 7 & 4 & 2 & 2 \\
\hline & P. direto & $8,6 \mathrm{a}$ & 4,0 & 16,4 & 32,8 & 172 & 43 & 19 & 11 & 7 & 5 \\
\hline \multirow[t]{3}{*}{$\mathrm{Fe}\left(\mathrm{mg} \mathrm{L}^{-1}\right)$} & Arado & $68,1 \mathrm{c}$ & 44,0 & 110,0 & 19,9 & 64 & 16 & 7 & 4 & 3 & 2 \\
\hline & Grade & $80,3 b$ & 58,0 & 143,0 & 17,2 & 47 & 12 & 5 & 3 & 2 & 1 \\
\hline & P. direto & $89,9 a$ & 65,0 & 115,0 & 12,1 & 23 & 6 & 3 & 1 & 1 & 1 \\
\hline \multirow[t]{3}{*}{$\operatorname{Mn}\left(\mathrm{mg} \mathrm{L}^{-1}\right)$} & Arado & $11,5 b$ & 8,0 & 15,0 & 13,4 & 29 & 7 & 3 & 2 & 1 & 1 \\
\hline & Grade & $11,5 b$ & 9,0 & 15,0 & 9,9 & 16 & 4 & 2 & 1 & 1 & 1 \\
\hline & P. direto & $15,5 \mathrm{a}$ & 9,0 & 21,0 & 15,9 & 41 & 10 & 5 & 3 & 2 & 1 \\
\hline \multirow[t]{3}{*}{$\mathrm{B}\left(\mathrm{mg} \mathrm{L}^{-1}\right)$} & Arado & $1,0 \mathrm{~b}$ & 0,6 & 1,5 & 19,8 & 63 & 16 & 7 & 4 & 3 & 2 \\
\hline & Grade & $1,3 \mathrm{a}$ & 0,8 & 1,8 & 20,0 & 64 & 16 & 8 & 4 & 3 & 2 \\
\hline & P. direto & $1,1 \mathrm{~b}$ & 0,6 & 1,8 & 23,8 & 91 & 23 & 11 & 6 & 4 & 3 \\
\hline \multirow[t]{3}{*}{$\mathrm{MO}\left(\mathrm{g} \mathrm{kg}^{-1}\right)$} & Arado & $15,4 \mathrm{c}$ & 13,0 & 18,0 & 8,8 & 12 & 3 & 1 & 1 & 1 & 1 \\
\hline & Grade & $17,2 b$ & 15,0 & 19,0 & 6,7 & 7 & 2 & 1 & 1 & 1 & 1 \\
\hline & P. direto & $18,2 \mathrm{a}$ & 15,0 & 22,0 & 7,9 & 10 & 3 & 2 & 1 & 1 & 1 \\
\hline \multirow[t]{3}{*}{ Argila $\left(\mathrm{g} \mathrm{kg}^{-1}\right)$} & Arado & $347,0 \mathrm{a}$ & 295,0 & 415,0 & 8,6 & 12 & 3 & 2 & 1 & 1 & 1 \\
\hline & Grade & $314,0 \mathrm{~b}$ & 300,0 & 420,0 & 8,0 & 11 & 3 & 2 & 1 & 1 & 1 \\
\hline & P. direto & $356,0 \mathrm{a}$ & 300,0 & 420,0 & 8,2 & 11 & 3 & 2 & 1 & 1 & 1 \\
\hline
\end{tabular}

(1)Valores seguidos pela mesma letra não diferem pelo teste t, a $5 \%$ de probabilidade. 
dade de 5-20 cm, apresentou maior coeficiente de variação em relação aos tratamentos arado e grade, e sobressaíram aos coeficientes apresentados na profundidade de $0-5 \mathrm{~cm}$ nos respectivos tratamentos. Os altos coeficientes de variação encontrados em ambas as camadas, nos três tratamentos, mostram a alta variabilidade de distribuição deste elemento no solo. No plantio direto, embora não haja revolvimento uniformizador do solo, as adubações sucessivas na mesma área, em locais quase nunca coincidentes, após alguns anos conduziriam a uma homogeneização em relação aos teores dos nutrientes do solo (Souza, 1992). Porém, com os altos valores do coeficiente de variação, não foi observada esta homogeneização, e sim, alta variabilidade no tratamento plantio direto, talvez pelo pouco tempo de utilização desse sistema na área experimental.

$\mathrm{O}$ Fe encontra-se amplamente distribuído nos perfis da maioria dos solos, alcançando, em alguns casos, quantidades muito altas na camada arável (Bataglia, 1991). Os valores médios de Fe no tratamento plantio direto foram os maiores, embora na profundidade de 5-20 cm, os valores dos tratamentos grade e plantio direto não tenham diferido signi- ficativamente entre si (Tabelas 1 e 2). Couto \& Klamt (1999), utilizando o preparo convencional sob pivô central, constataram que o Fe, $\mathrm{Mn}, \mathrm{B}$ e Cu apresentaram valores abaixo dos respectivos níveis críticos, e verificaram que as operações de preparo parecem não ter sido eficientes para distribuir e homogeneizar os fertilizantes no solo. Os maiores valores máximos e mínimos de Fe ocorreram no tratamento grade, em ambas profundidades. De modo geral, o valor do coeficiente de variação apresentado pelo Fe, em todos os tratamentos, nas duas profundidades, foram relativamente mais baixos em relação aos de $\mathrm{Zn}$. O Fe apresenta uma distribuição constante e uniforme no solo, com todos os valores se situando dentro da faixa de média disponibilidade, de 31 a $200 \mathrm{mg} \mathrm{L}^{-1}$ de Fe (Haag et al., 1982).

Os valores médios de Mn na primeira camada nos tratamentos arado e grade foram diferentes em relação ao tratamento plantio direto; o mesmo ocorreu na segunda camada. Nas duas profundidades, os valores de Mn foram muito semelhantes nos tratamentos arado e grade. O maior valor de Mn no plantio direto na primeira camada revela que houve concentração do elemento na superfície do solo.

Tabela 2. Valores médios, mínimos, máximos, coeficiente de variação (CV) e número mínimo de subamostras necessário para estimar os teores de $\mathrm{Cu}, \mathrm{Zn}, \mathrm{Fe}, \mathrm{Mn}, \mathrm{B}$, matéria orgânica (MO) e argila do solo, para várias porcentagens de variação em torno da média, nos tratamentos arado, grade e plantio direto, na profundidade de 5-20 cm de solo.

\begin{tabular}{|c|c|c|c|c|c|c|c|c|c|c|c|}
\hline \multirow[t]{2}{*}{ Variável } & \multirow[t]{2}{*}{ Tratamento } & \multirow[t]{2}{*}{ Média $^{(1)}$} & \multirow[t]{2}{*}{ Mínimo } & \multirow[t]{2}{*}{ Máximo } & \multirow[t]{2}{*}{$\mathrm{CV}$} & \multicolumn{6}{|c|}{ Número de subamostras para variação em torno da média } \\
\hline & & & & & & $5 \%$ & $10 \%$ & $15 \%$ & $20 \%$ & $25 \%$ & $30 \%$ \\
\hline \multirow[t]{3}{*}{$\mathrm{Cu}\left(\mathrm{mg} \mathrm{L}^{-1}\right)$} & Arado & $2,5 b$ & 1,8 & 6,2 & 31,7 & 161 & 40 & 18 & 10 & 6 & 4 \\
\hline & Grade & $3,1 \mathrm{a}$ & 2,2 & 14,9 & 63,5 & 645 & 161 & 72 & 40 & 26 & 18 \\
\hline & P. direto & $3,3 a$ & 2,0 & 15,4 & 70,1 & 787 & 197 & 87 & 49 & 31 & 22 \\
\hline \multirow[t]{3}{*}{$\overline{\mathrm{Zn}\left(\mathrm{mg} \mathrm{L}^{-1}\right)}$} & Arado & $4,7 b$ & 1,3 & 13,6 & 45,1 & 325 & 81 & 36 & 20 & 13 & 9 \\
\hline & Grade & $6,9 a$ & 3,8 & 24,7 & 48,5 & 377 & 94 & 42 & 24 & 15 & 10 \\
\hline & P. direto & $6,6 \mathrm{ab}$ & 2,2 & 44,0 & 100,9 & 1.631 & 408 & 181 & 102 & 65 & 45 \\
\hline \multirow[t]{3}{*}{$\overline{\mathrm{Fe}}\left(\mathrm{mg} \mathrm{L}^{-1}\right)$} & Arado & $81,5 \mathrm{~b}$ & 39,0 & 132,0 & 27,7 & 123 & 31 & 14 & 8 & 5 & 3 \\
\hline & Grade & $106,9 a$ & 68,0 & 176,0 & 25,9 & 107 & 27 & 12 & 7 & 4 & 3 \\
\hline & P. direto & $109,8 \mathrm{a}$ & 71,0 & 154,0 & 18,1 & 52 & 13 & 6 & 3 & 2 & 1 \\
\hline \multirow[t]{3}{*}{$\operatorname{Mn}\left(\mathrm{mg} \mathrm{L}^{-1}\right)$} & Arado & $11,7 \mathrm{a}$ & 9,0 & 18,0 & 15,7 & 40 & 10 & 4 & 2 & 2 & 1 \\
\hline & Grade & $10,9 a$ & 1,0 & 20,0 & 21,6 & 75 & 19 & 8 & 5 & 3 & 2 \\
\hline & P. direto & $9,8 \mathrm{~b}$ & 5,0 & 19,0 & 28,0 & 126 & 31 & 14 & 8 & 5 & 4 \\
\hline \multirow{3}{*}{$\mathrm{B}\left(\mathrm{mg} \mathrm{L}^{-1}\right)$} & Arado & $1,1 \mathrm{c}$ & 0,5 & 1,8 & 22,3 & 80 & 20 & 9 & 5 & 4 & 3 \\
\hline & Grade & $1,4 \mathrm{a}$ & 0,8 & 2,2 & 23,2 & 86 & 22 & 10 & 6 & 4 & 3 \\
\hline & P. direto & $1,3 \mathrm{~b}$ & 0,6 & 2,1 & 19,9 & 91 & 16 & 8 & 4 & 3 & 2 \\
\hline \multirow[t]{3}{*}{$\mathrm{MO}\left(\mathrm{g} \mathrm{kg}^{-1}\right)$} & Arado & $15,4 a$ & 12,0 & 20,0 & 10,9 & 19 & 5 & 2 & 1 & 1 & 1 \\
\hline & Grade & $15,7 \mathrm{a}$ & 19,0 & 19,0 & 6,6 & 7 & 2 & 1 & 1 & 1 & 1 \\
\hline & P. direto & $15,4 \mathrm{a}$ & 13,0 & 18,0 & 6,2 & 6 & 2 & 1 & 1 & 1 & 1 \\
\hline \multirow[t]{3}{*}{ Argila $\left(\mathrm{g} \mathrm{kg}^{-1}\right)$} & Arado & $349,0 \mathrm{~b}$ & 295,0 & 405,0 & 25,6 & 9 & 3 & 1 & 1 & 1 & 1 \\
\hline & Grade & $384,0 \mathrm{a}$ & 305,0 & 430,0 & 32,3 & 12 & 3 & 2 & 1 & 1 & 1 \\
\hline & P. direto & $385,0 \mathrm{a}$ & 325,0 & 425,0 & 26,0 & 7 & 2 & 1 & 1 & 1 & 1 \\
\hline
\end{tabular}

${ }^{(1)}$ Valores seguidos pela mesma letra não diferem pelo teste t, a $5 \%$ de probabilidade. 
A amplitude encontrada nos tratamentos, nas duas camadas, foi relativamente baixa, se comparada aos valores encontrados para $\mathrm{Cu}, \mathrm{Zn}$ e $\mathrm{Fe}$. Isto pode ser comprovado pelos baixos coeficientes de variação. Assim, pode-se afirmar que houve pouca variabilidade deste elemento na área.

A disponibilidade de B no solo pode ser influenciada pelo cultivo mínimo, plantio direto, rotação de culturas e adubação verde que, por propiciarem maior controle da mineralização da matéria orgânica e a reciclagem de nutrientes dos horizontes subsuperficiais para o superficial podem regular melhor o nível de B no solo (Dantas, 1991). O maior valor médio de $\mathrm{B}$ foi encontrado, nas duas camadas, no tratamento grade. $\mathrm{O}$ valor médio de $\mathrm{B}$ foi maior na profundidade de 5-20 $\mathrm{cm}$.

A presença da matéria orgânica no solo está associada com a disponibilidade, a quantidade e a retenção de alguns micronutrientes no solo, como B, Cu e Zn (Dantas, 1991; Ferreira \& Cruz, 1991; Souza \& Ferreira, 1991). Deficiências de Fe, Cu, Mn e, ou $\mathrm{Zn}$ têm sido verificadas em solos com alto teor de matéria orgânica, em decorrência da sua ação quelante sobre esses íons; à medida que ela se decompõe, ocorre a liberação dos micronutrientes (Oliveira et al., 1998). O teor de matéria orgânica diferiu entre os tratamentos apenas na primeira camada. $\mathrm{O}$ plantio direto apresentou o maior valor médio, por causa da manutenção dos restos da cultura anterior na superfície e do pouco revolvimento do solo, facilitando o acúmulo da matéria orgânica pela decomposição da palhada, reciclando nutrientes. Solos com uso do cultivo mínimo e do plantio direto podem, ao longo do tempo, aumentar o teor de matéria orgânica do solo (Thung \& Oliveira, 1998). Os maiores coeficientes de variação encontrados, em ambas profundidades, foram obtidos no tratamento com preparo de solo com arado, embora esta variabilidade não tenha sido tão alta. Souza (1992) também observou baixo coeficiente de variação da matéria orgânica, menor que $14 \%$, no sistema plantio direto, onde, devido à não-incorporação dos resíduos culturais, esperavase maior variabilidade.

Com relação ao teor de argila, na primeira camada, os tratamentos arado e plantio direto apresentaram significativamente maiores valores do que o tratamento grade, enquanto na segunda camada, os maiores valores foram obtidos nos tratamentos grade e plantio direto. Normalmente, quanto maior o teor de argila do solo mais alto é o teor de matéria orgânica (Thung \& Oliveira, 1998). Os coeficientes de variação observados mostraram baixa variabilidade. De acordo com Souza (1992), trata-se de uma característica do solo pouco influenciada pelo cultivo, principalmente quando estudada em diferentes profundidades. Por não haver grandes diferenças nos teores de argila, não são passíveis de causar aumento na variabilidade por inversão de camadas, quando das operações de preparo do solo.

Uma das utilidades do coeficiente de variação em estudos de variabilidade do solo é permitir calcular o número mínimo de subamostras para estimar o valor de uma característica em determinada área, com exatidão preestabelecida (Souza, 1992). Assim, usandose a fórmula descrita por Cline (1944), calculou-se o número mínimo de amostras, para estimar os teores de $\mathrm{Cu}, \mathrm{Zn}, \mathrm{Fe}, \mathrm{Mn}, \mathrm{B}, \mathrm{MO}$ e argila do solo, com diferentes porcentagens de variação em torno da média $(5,10,15,20,25$ e $30 \%)$, em todos os tratamentos (Tabelas 1 e 2). Em relação ao $\mathrm{Cu}$, dado o alto coeficiente de variação, o número de subamostras necessárias para obter uma variação de $5 \%$ em torno da média é grande, cerca de 367 subamostras no tratamento plantio direto na camada de $0-5 \mathrm{~cm}$, e de 787 subamostras na camada de 5-20 cm, inexequíivel na prática. Quantidades inexeqüíveis de subamostras foram encontradas por Forsythe (1970), Barreto et al. (1974) e Souza (1992) para estimar os valores de P e de K do solo para obter uma variação de 5-10\% em torno do valor médio.

Utilizando-se o procedimento de coletar 20 subamostras para compor uma amostra composta, como proposto pela Comissão de Fertilidade do Solo do Estado de Minas Gerais (1989), para o Estado de Minas Gerais e pela Comissão de Fertilidade do SoloRS/SC (1995), para Santa Catarina e Rio Grande do $\mathrm{Sul}$, os resultados obtidos para o $\mathrm{Cu}$ mostram que para a profundidade de $0-5 \mathrm{~cm}$ e para os tratamentos arado e grade, os teores de $\mathrm{Cu}$ estariam sendo estimados, com uma variação em torno da média, menor que $10 \%$. Para o plantio direto, nesta mesma profundidade, o erro seria superior a $20 \%$. Na profundidade de 5-20 $\mathrm{cm}$ o erro de estimativa estaria em torno de $15 \%$ no tratamento arado, chegando a $30 \%$ nos tratamentos grade e plantio direto. 
De acordo com Souza (1992), a importância do erro de amostragem na estimativa do valor de uma característica do solo, devido ao número de subamostras utilizado, está em função do valor esperado para aquela característica. Assim, quando se espera obter o teor de um nutriente no solo, acima do nível crítico, pode-se admitir maior desvio porcentual da média, resultante de um baixo número de subamostras, já que isto pouco influenciará na decisão da adubação. Mas, se os valores esperados estão abaixo do nível crítico, uma estimativa mais precisa deve ser buscada, procurando coletar maior número de subamostras, pois erros de estimativas poderão deslocar o valor obtido para outra faixa de recomendação e provocar adubações maiores ou menores que as necessárias.

Em relação ao Zn, na profundidade de $0-5 \mathrm{~cm}$, encontrou-se um número mínimo de subamostras inexequíivel na prática, para uma variação de $5 \%$ em torno da média. Para o teor de Zn ser estimado com este erro seriam necessários, respectivamente, para os tratamentos arado, grade e plantio direto, cerca de 449, 62 e 172 subamostras. Na profundidade de 5-20 cm, o número de subamostras é ainda maior.

Quanto ao Fe, Mn e B, o erro em torno do valor médio, de uma amostra representativa de 20 subamostras, estaria em torno de $10 \%$, indicando que estes nutrientes são avaliados com boa precisão se são seguidas as recomendações da amostragem do solo da Comissão de Fertilidade de Solos de Goiás (1988) e da Comissão de Fertilidade do Solo do Estado de Minas Gerais (1989).

Maior precisão ainda, ou seja, erro menor que 5\% em torno do valor médio, é conseguida para a estimativa dos teores de matéria orgânica e de argila, com a análise de uma amostra de solo composta de 20 subamostras. Isto é possível graças à baixa variabilidade dessas características do solo. Souza (1992) verificou que o número recomendado de 10 a 15 subamostras estaria estimando a matéria orgânica e a argila com uma variação em torno da média inferior a $5 \%$.

A distribuição espacial dos valores de $\mathrm{Zn}$ do solo nos sistemas de preparo e nas duas profundidades está apresentada na Figura 1. Os valores foram agrupados nas faixas de teor de $\mathrm{Zn}$ de até $4,0 \mathrm{mg} \mathrm{L}^{-1}$ (baixa), 4,1-6,0 $\mathrm{mg} \mathrm{L}^{-1}$ (média), e 6,1-40,0 $\mathrm{mg} \mathrm{L}^{-1}$ (alta), de acordo com Costa et al. (1984). Por convenção, estabeleceram-se as cores vermelho, azul e verde para representar, respectivamente, as faixas de disponibilidade baixa, média e alta.

No sistema plantio direto, os maiores valores de $\mathrm{Zn}$ (cor verde) encontram-se na camada de $0-5 \mathrm{~cm}$ em relação à 5-20 cm, caracterizando maior variabilidade do nutriente em profundidade, dentro das faixas preestabelecidas. Entretanto, o tratamento apresentou menor variabilidade horizontal, identificada pela maior uniformidade das cores na Figura 1. No tratamento arado, houve menor variabilidade dos
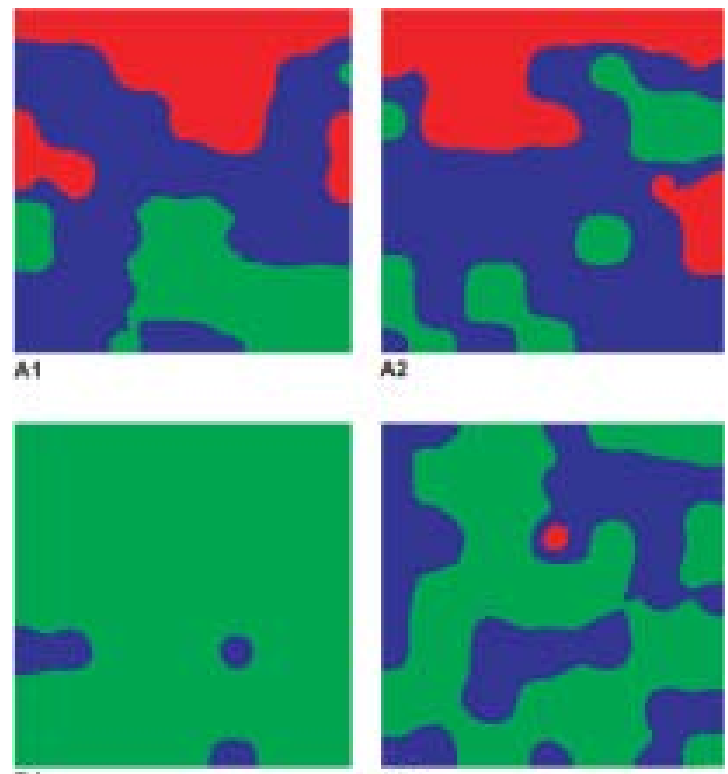

B1
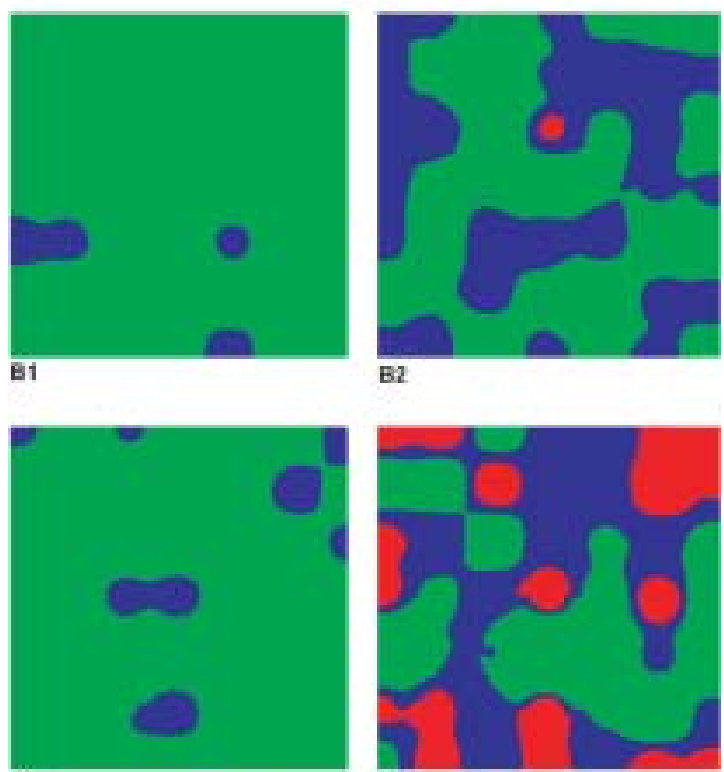

c1
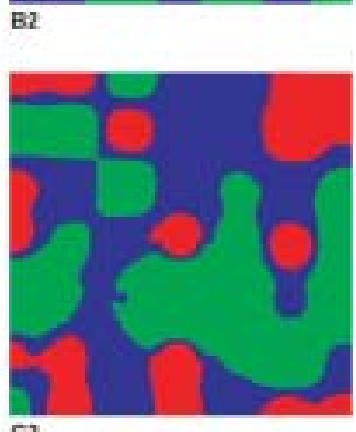

C2

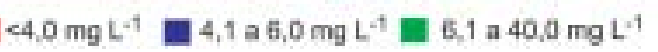

Figura 1. Distribuição espacial dos teores de zinco do solo ( $\left.\mathrm{mg} \mathrm{L}^{-1}\right)$ nos diferentes sistemas de preparo (A: arado; $\mathrm{B}$ : grade; C: plantio direto) e profundidade de amostragem (1: $0-5 \mathrm{~cm} ; 2: 5-20 \mathrm{~cm})$. 
valores de $\mathrm{Zn}$ entre as duas camadas de solo, provavelmente pela ação uniformizadora do implemento, no processo de revolvimento dessas camadas.

No caso da MO, a Comissão de Fertilidade do Solo do Estado de Minas Gerais (1989) descreve a disponibilidade no solo de $0-15 \mathrm{~g} \mathrm{~kg}^{-1}$ como baixa, 16-30 $\mathrm{g} \mathrm{kg}^{-1}$, média e maior de $30 \mathrm{~g} \mathrm{~kg}^{-1}$, alta. Também, por convenção, estabeleceram-se as cores vermelho e azul para representar, respectivamente, as faixas de disponibilidade baixa e média (Figura 2). Não foram observados valores de MO do solo na faixa de disponibilidade alta, indicando que mesmo o

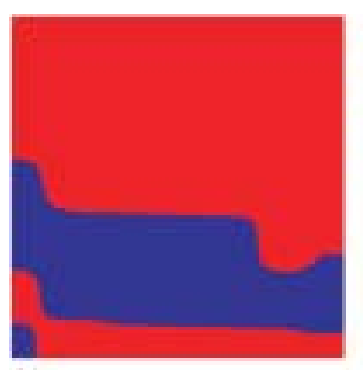

A1
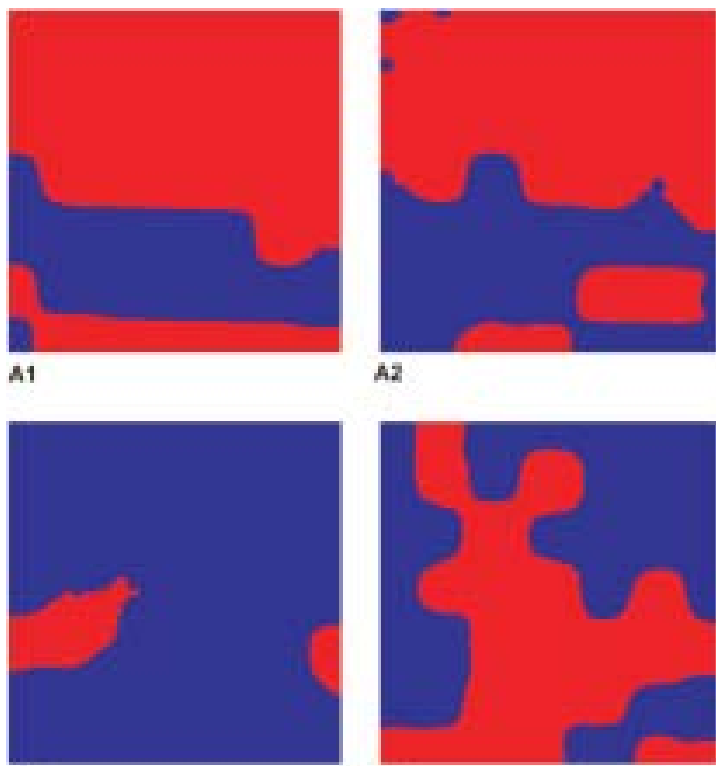

日1
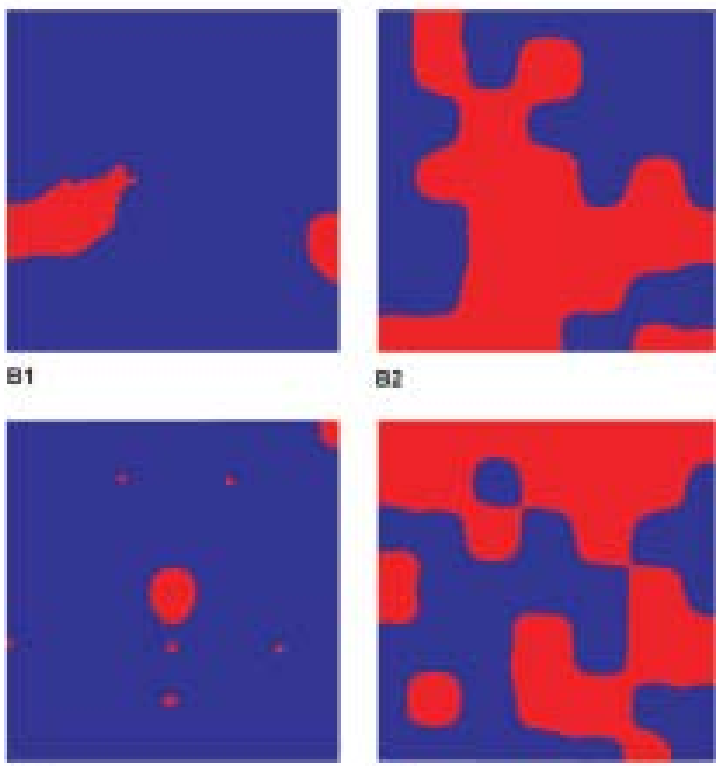

C1

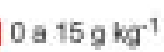

B2

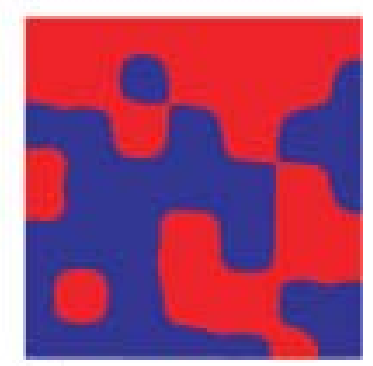

c2

plantio direto, após cinco anos de cultivo, não elevou o teor de MO. Semelhante ao ocorrido com o Zn, no sistema plantio direto os maiores valores de MO do solo encontram-se na camada de 0-5 cm em relação à 5-20 cm, caracterizando maior variabilidade deste atributo em profundidade. Por outro lado, no tratamento arado, houve maior uniformidade dos valores entre as duas camadas de solo e menores valores de matéria orgânica.

\section{Conclusões}

1. Os valores médios de $\mathrm{Cu}, \mathrm{Zn}, \mathrm{Fe}, \mathrm{Mn}, \mathrm{B}$, matéria orgânica e argila variam conforme o sistema de preparo e a profundidade do solo.

2. Na camada de $0-5 \mathrm{~cm}$, os valores de $\mathrm{Cu}, \mathrm{Mn}, \mathrm{B}$, matéria orgânica e argila são maiores no sistema plantio direto do que nos tratamentos com arado de aiveca e com grade aradora.

3. Os valores de $\mathrm{Cu}$ e de $\mathrm{Zn}$ apresentam as maiores variabilidades, e os de matéria orgânica e argila, as menores.

4. Utilizando-se o procedimento de coletar 20 subamostras para formar uma amostra composta, os teores de argila, matéria orgânica, B e Mn são estimados com erro em torno de $10 \%$ do valor médio, e os de $\mathrm{Cu}$ e $\mathrm{Zn}$, com erro superior a $25 \%$.

\section{Referências}

BARRETO, A. C.; NOVAIS, R. F.; BRAGA, J. M. Determinação estatística do número de amostras simples de solo para avaliação de sua fertilidade. Revista Ceres, Viçosa, v. 21, n. 114, p. 142-147, 1974.

BATAGLIA, O. C. Ferro. In: FERREIRA, M. E.; CRUZ, M. C. P. da (Ed.). Micronutrientes na agricultura. Piracicaba: Potafos, 1991. p. 159-172.

CLINE, M. G. Principles of soil sampling. Soil Science, Baltimore, v. 58, n. 4, p. 275-288, 1944.

COMISSÃO DE FERTILIDADE DE SOLOS DE GOIÁS (Goiânia, GO). Recomendações de corretivos e fertilizantes para Goiás: 5a aproximação. Goiânia: UFG/ Emgopa, 1988. 101 p. (Informativo Técnico, 1).

Figura 2. Distribuição espacial dos teores de matéria orgânica do solo $\left(\mathrm{g} \mathrm{kg}^{-1}\right)$ nos sistemas de preparo (A: arado; B: grade; C: plantio direto) e profundidade de amostragem (1: $0-5 \mathrm{~cm} ; 2: 5-20 \mathrm{~cm})$.

COMISSÃO DE FERTILIDADE DO SOLO DO ESTADO DE MINAS GERAIS (Lavras, MG). Recomenda- 
ções para o uso de corretivos e fertilizantes em Minas Gerais: 4⿳亠口冋口 aproximação. Lavras: Epamig, 1989. 176 p.

COMISSÃO DE FERTILIDADE DO SOLO-RS/SC (Passo Fundo, RS). Recomendações de adubação e calagem para os Estados do Rio Grande do Sul e Santa Catarina. 3. ed. Passo Fundo: Sociedade Brasileira de Ciência do Solo, 1995. $223 \mathrm{p}$.

COSTA, A. C. M.; DI PIETRO, C. D.; FERNANDES, D. R.; GROHMANN, F.; LACERDA, L. A. O.; SCALI, M. H.; COSTA, P. C.; SANTINATO, R. Levantamento nutricional de cafeeiro no Estado de São Paulo pelas análises de solo e foliar. In: CONGRESSO BRASILEIRO DE PESQUISAS CAFEEIRAS, 11., 1984, Londrina. Anais... Rio de Janeiro: Instituto Brasileiro do Café, 1984. p. 298-303.

COUTO, E. G.; KLAMT, E. Variabilidade espacial de micronutrientes em solo sob pivô central no sul do Estado de Mato Grosso. Pesquisa Agropecuária Brasileira, Brasília, v. 34, n. 12, p. 2321-2329, dez. 1999.

DANTAS, J. P. Boro. In: FERREIRA, M. E.; CRUZ, M. C. P. da. (Ed.). Micronutrientes na agricultura. Piracicaba: Potafos, 1991. p. 113-130.

EMBRAPA. Centro Nacional de Pesquisa de Solos (Rio de Janeiro, RJ). Manual de métodos de análise de solo. 2. ed. Rio de Janeiro, 1997. 212 p. (Documentos, 1).

FERREIRA, M. E.; CRUZ, M. C. P. da. Cobre. In: (Ed.). Micronutrientes na agricultura. Piracicaba: Potafos, 1991. p. 131-157.

FORSYTHE, W. M. Importancia de la variabilidad de las propiedades del suelo para evaluarlas en su manejo. Turrialba, San José, v. 20, n. 4, p. 445-451, 1970.

HAAG, H. P.; MINAME, K.; DECHEN, A. R. Micronutrientes em hortaliças. Campinas: Fundação Cargill, 1982. $26 \mathrm{p}$.
HOROWITZ, A.; DANTAS, H. da S. Geoquímica dos elementos menores nos solos de Pernambuco. III. Cobre na zona Litoral-Mata. Pesquisa Agropecuária Brasileira: Série Agronomia, Rio de Janeiro, v. 8, n. 7, p. 169-176, 1973.

INSTITUTO NACIONAL DE PESQUISAS ESPACIAIS (São José dos Campos, SP). Manual de usuário do SGI. São José dos Campos, 1990. 1 v.

OLIVEIRA, I. P. de; KLUTHCOUSKI, J.; BALBINO, L. C.; BUSO, L. H.; YOKOYAMA, L. P.; MAGNABOSCO, C. de U.; SCARPATI, M. T. V. Sistema Barreirão: emprego de micronutrientes na recuperação de pastagens. Santo Antônio de Goiás: EmbrapaCNPAF, 1998. 36 p. (Circular Técnica, 30).

RAIJ, B. van. Fertilidade do solo e adubação. São Paulo: Agronômica Ceres, 1991. 343 p.

RAIJ, B. van; BATAGLIA, O. C. Análise química do solo. In: FERREIRA, M. E.; CRUZ, M. C. P. da (Ed.). Micronutrientes na agricultura. Piracicaba: Potafos, 1991. p. 333-355.

SANTOS, H. L.; VASCONCELLOS, C. A. Determinação do número de amostras de solo para análise química em diferentes condições de manejo. Revista Brasileira de Ciência do Solo, Campinas, v. 11, n. 2, p. 97-100, 1987.

SOUZA, E. C. A. de; FERREIRA, M. E. Zinco. In: FERREIRA, M. E.; CRUZ, M. C. P. da (Ed.). Micronutrientes na agricultura. Piracicaba: Potafos, 1991. p. 219-242.

SOUZA, S. L. da. Variabilidade espacial do solo em sistema de manejo. Porto Alegre: UFRGS, 1992. $162 \mathrm{f}$. Tese (Doutorado em ciência do solo) - Universidade Federal do Rio Grande do Sul, Porto Alegre.

ThUnG, M. D. T.; OliVeirA, I. P. de. Problemas abióticos que afetam a produção do feijoeiro e seus métodos de controle. Santo Antônio de Goiás: EmbrapaCNPAF, 1998. $172 \mathrm{p}$. 